\title{
Neuropathic pain and cytokines: current perspectives
}

\author{
This article was published in the following Dove Press journal: \\ Journal of Pain Research \\ 20 November 2013 \\ Number of times this article has been viewed
}

\author{
Anna K Clark \\ Elizabeth A Old \\ Marzia Malcangio \\ Wolfson Centre for Age Related \\ Diseases, King's College London, \\ London, UK
}

Correspondence: Marzia Malcangio; Anna K Clark

Wolfson Centre for Age Related Diseases, King's College London, Guy's Campus, London Bridge, London, SEI IUL, UK

Tel +44 2078486092

$\mathrm{Fax}+442078486165$

Email marzia.malcangio@kcl.ac.uk; anna.clark@kcl.ac.uk

\begin{abstract}
Neuropathic pain represents a major problem in clinical medicine because it causes debilitating suffering and is largely resistant to currently available analgesics. A characteristic of neuropathic pain is abnormal response to somatic sensory stimulation. Thus, patients suffering peripheral neuropathies may experience pain caused by stimuli which are normally nonpainful, such as simple touching of the skin or by changes in temperature, as well as exaggerated responses to noxious stimuli. Convincing evidence suggests that this hypersensitivity is the result of pain remaining centralized. In particular, at the first pain synapse in the dorsal horn of the spinal cord, the gain of neurons is increased and neurons begin to be activated by innocuous inputs. In recent years, it has become appreciated that a remote damage in the peripheral nervous system results in neuronal plasticity and changes in microglial and astrocyte activity, as well as infiltration of macrophages and T cells, which all contribute to central sensitization. Specifically, the release of pronociceptive factors such as cytokines and chemokines from neurons and non-neuronal cells can sensitize neurons of the first pain synapse. In this article we review the current evidence for the role of cytokines in mediating spinal neuron-non-neuronal cell communication in neuropathic pain mechanisms following peripheral nerve injury. Specific and selective control of cytokine-mediated neuronal-glia interactions results in attenuation of the hypersensitivity to both noxious and innocuous stimuli observed in neuropathic pain models, and may represent an avenue for future therapeutic intervention.
\end{abstract}

Keywords: anti-inflammatory cytokines, proinflammatory cytokines, microglia, astrocytes, first pain synapse

\section{Introduction}

Neuropathic pain is a chronic condition which arises following lesion or dysfunction of the somatosensory nervous system and may result in complex alterations in cognitive and emotional brain functions. Neuropathic pain commonly accompanies a variety of conditions, including peripheral nerve injury (postsurgical pain), central nervous system (CNS) injury (multiple sclerosis, spinal cord injury), viral infections (eg, postherpetic neuralgia), tumors, and metabolic disorders such as diabetes mellitus. In particular, chronic neuropathic pain resulting from peripheral nerve damage is a significant clinical problem which often proves refractory to current treatments, partially due to the fact that the mechanisms are insufficiently understood. Damage to a peripheral nerve results in amplification of responses to peripherally applied painful stimuli at the first synapse in the nociceptive pathway (first pain synapse), leading to excessive activity in the spinal cord. Traditionally, this phenomenon has been considered a purely neuronal response. However, extensive preclinical evidence now indicates a critical 
contribution of non-neuronal cells in the mechanisms that underlie neuropathic pain states, thereby providing novel therapeutic targets.

Specifically, following peripheral nerve trauma, nonneuronal cells at the site of injury and in the spinal cord begin to secrete a plethora of proinflammatory mediators that may modulate nociceptive function. In the injured peripheral nerve, the infiltration of both innate and adaptive immune cells is critical for the early initiation phase of neuropathic pain in rodent models. ${ }^{1,2}$ In the spinal cord, disruption of homeostasis causes resident glial cells (microglia and astrocytes) to transition into pain-related enhanced response states, ${ }^{3-5}$ characterized by morphological changes (Figure 1) and enhanced synthesis and release of algogenic substances. Additionally, remote nerve injury in the periphery results in an immediate and transient alteration of the blood-spinal cord barrier (BSCB) integrity in the lumbar enlargement, ${ }^{6,7}$ where injured fibers terminate and infiltration of peripheral immune cells, such as macrophages ${ }^{6,8,9}$ and T lymphocytes, ${ }^{8,10-12}$ occurs into the dorsal horn (Figure 1).

Understanding the sequence and nature of the events that govern neuroimmune communication is critical for the discovery of new mechanisms and targets for neuropathic pain treatment. In particular, cytokines are receiving growing interest as modulators of neuronal plasticity and enhanced nociceptive transmission under conditions of neuropathic pain. Here we review the evidence in relation to the spinal cord mechanisms of a select number of cytokines subsequent to peripheral nerve injury.

Cytokines are pivotal mediators in the multistep response that the host organizes to counteract foreign insults; they drive the innate immune response and are critical for survival of the host organism. The cytokines are small intracellular polypeptides (5-140 kDa) which are subdivided into a number of large families. For example, the Interleukin (IL) family constitutes over 30 members. They are generally synthesized as larger size precursors which are proteolytically cleaved to produce the active form. The cytokines, being nonstructural proteins, are classified on the basis of their biological activity as proinflammatory (eg, IL-1 family) or anti-inflammatory (IL-10 family) cytokines. They are effective at very small concentrations (pM) and perform various biological functions in immunology and inflammation which depend on the cell type expressing their receptors. ${ }^{13}$

\section{Proinflammatory cytokines and spinal mechanisms in neuropathic pain}

\section{IL-I $\beta$}

IL-1 $\beta$ is a small $(17.5 \mathrm{kDa})$ neutral proinflammatory cytokine belonging to the IL-1 gene family. IL-1 $\beta$ is the prototypical

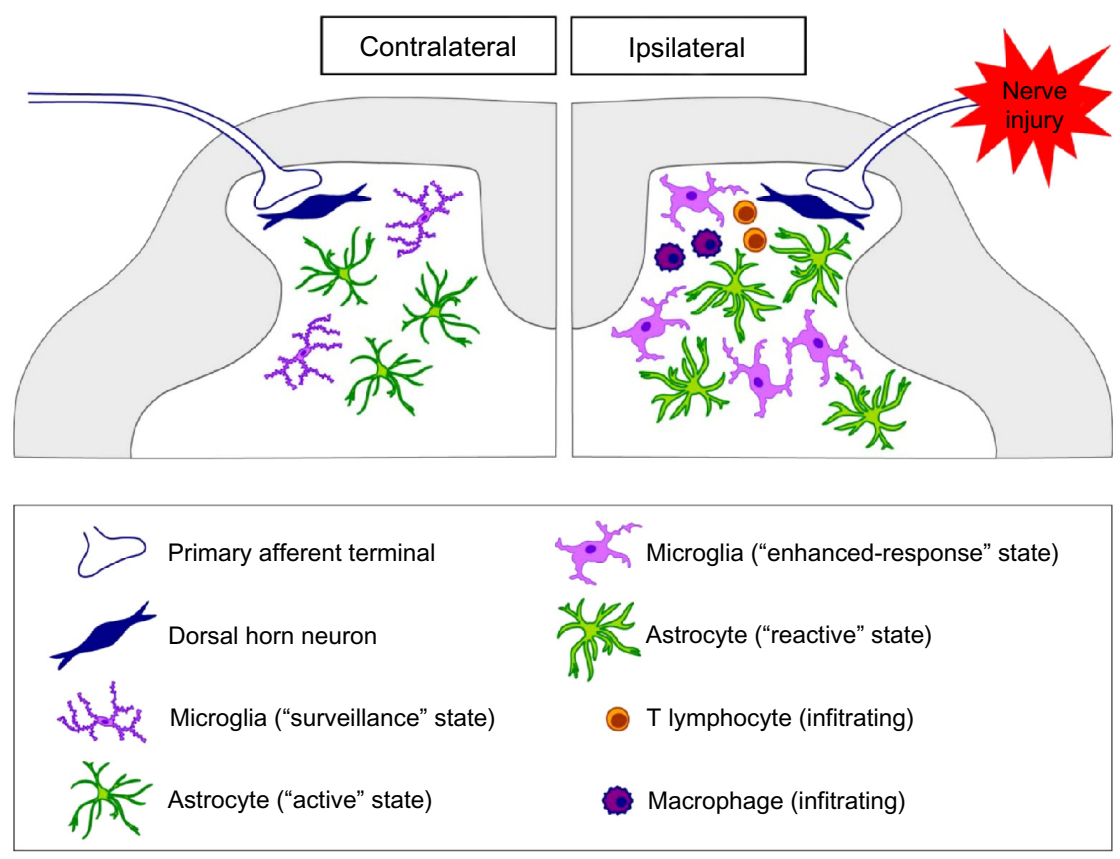

Figure I Schematic representation of morphological glial cell changes and immune cell infiltration in the lumbar spinal cord following peripheral nerve injury. Notes: The altered activity states of spinal glial cells induced by peripheral nerve injury are most commonly identified by changes in cell morphology. Microglia transition from a surveillant state to an "enhance-response" state, ${ }^{3,4}$ which is evident by retraction of their fine processes and enlargement of cell bodies. Astrocytes transition from an active state to reactive state. ${ }^{3,5}$ Infiltration of macrophages and T lymphocytes is also evident within the dorsal horn. ${ }^{6,8-12}$ 
multifunctional cytokine, having the ability to induce the expression of other proinflammatory mediators, and is central to setting in motion the host's inflammatory and immune responses. IL-1 $\beta$ has a range of biological effects that reflect the expression of the IL-1 receptor 1 (IL-1R1) on target cell types.

IL-1 $\beta$ was one of the first cytokines to be implicated in peripheral nerve injury-induced neuropathic pain mechanisms in rodents. Genetic impairment of IL-1 $\beta$ signaling attenuates nerve injury-induced pain behaviors. ${ }^{14,15}$ Thus, mice lacking both IL- $1 \beta$ and IL- $1 \alpha$ show a significant reduction in mechanical hypersensitivity in two models of peripheral nerve injury (spinal nerve ligation [SNL] and chronic constriction of the sciatic nerve) ${ }^{14}$ Furthermore, both IL-1R1 null mice as well as IL-1 receptor antagonist (IL-1 ra) overexpressing mice fail to develop mechanical or thermal pain behaviors following SNL, ${ }^{15}$ suggesting a critical contribution of IL-1 signaling to nerve injury-induced pain. However, in both these genetic studies IL-1 signaling is impaired globally, making it difficult to determine the specific contribution of spinal signaling to the phenotype of these mice. Pharmacological studies have, however, delineated the role of spinal IL-1 $\beta$ in neuropathic pain states. Chronic intrathecal delivery of IL-1ra to mice is able to prevent the development of nerve injury-induced pain behaviors, and a single intrathecal administration of IL-1ra 4 days following injury is sufficient to reverse established mechanical hypersensitivity. ${ }^{16}$ Likewise, a single intrathecal administration of IL-1ra in the rat at either 10 days ${ }^{17}$ or 8 weeks ${ }^{18}$ following chronic constriction of the sciatic nerve is able to transiently reverse neuropathic hypersensitivity. Chemotherapy-induced neuropathy following paclitaxel is also transiently reversed by a single intrathecal administration of IL-1ra. ${ }^{19}$ Conversely, in a different model of peripheral nerve injury (SNL), chronic intrathecal treatment with IL$1 \mathrm{ra}$ in the rat is insufficient to prevent the development of nerve injury-induced pain behaviors; the coadministration of an inhibitor of tumor necrosis factor $\alpha$ (TNF $\alpha)$ signaling is required for significant attenuation of mechanical hypersensitivity. ${ }^{20}$ Intrathecal delivery of the IL-1 receptor antagonist is also able to prevent hypersensitivity associated with acute microglial activation following intrathecal lipopolysaccharide (LPS), ${ }^{21}$ or the HIV protein gp $120 .{ }^{22}$

Under physiological conditions, IL-1 $\beta$ is expressed at low levels in the spinal cord. Following peripheral nerve injury, spinal IL-1 $\beta$ expression is upregulated in a range of cell types. ${ }^{23-28}$ Although IL-1 $\beta$ is largely produced and secreted by glial cells, ${ }^{23-26}$ neuronal expression is also observed following models of peripheral neuropathy. ${ }^{27,28}$ The expression of the IL-1R1 in the spinal cord is also widespread, with neurons, microglia, and astrocytes all exhibiting receptor expression. ${ }^{24,26,29}$ One recent study utilized a pioneering in vivo spinal microdialysis technique to monitor cytokine levels in the cerebrospinal fluid (CSF) of rats following peripheral nerve injury. ${ }^{30}$ In neuropathic rats, activation of primary afferent fibers leads to increased release of IL-1 $\beta$ compared to sham animals, with neuronal-glial communication critical for this activity-dependent release. ${ }^{30}$ Indeed, in humans, patients with a range of painful peripheral neuropathies exhibit enhanced IL-1 $\beta$ levels (compared to normal controls) in their CSF. ${ }^{31,32}$

The signaling of many inflammatory mediators is regulated by proteases. Indeed, many of the proinflammatory agents that may modulate nociceptive transmission also require proteolytic processing in order for signal transduction to take place. IL-1 $\beta$ is synthesized as a $31 \mathrm{kDa}$ biologically inactive precursor (pro-IL-1 $\beta$ ). The maturation and release of IL-1 $\beta$ from immune cells, including microglia, is a tightly regulated process, requiring the cleavage of pro-IL-1 $\beta$ into the biologically active cytokine. The inflammasome is a caspase-activating complex comprising a scaffold of interacting proteins, which upon oligomerization induces activation of pro-caspase 1 , initiating processing of pro-IL-1 $\beta .{ }^{33}$ Caspase 1 is critical for the regulation of IL- $1 \beta$ maturation, but itself requires proteolytic activation. ${ }^{34}$ Indeed, several components of the inflammasome, including caspase 1, are upregulated in spinal microglia following peripheral nerve injury. ${ }^{35}$ Accordingly, spinal inhibition of caspase 1 effectively attenuates hypersensitivity following both peripheral nerve injury ${ }^{35}$ and intrathecal LPS, ${ }^{21}$ via reduced secretion of IL- $1 \beta$ from spinal microglia. ${ }^{21}$ The role of other components of the inflammasome in neuropathic pain is currently less clear.

The release of neuronal and astrocytic IL-1 $\beta$ under conditions of peripheral nerve injury have recently been attributed to matrix metalloproteases (MMPs). ${ }^{36}$ Nerve injury results in the enhanced activity of MMP9 and MMP2, leading to cleavage of pro-IL-1 $\beta$ in neurons and astrocytes, respectively. Accordingly, inhibition of either MMP9 or MMP2 is sufficient to reverse established neuropathic pain behaviors, via a reduction in biologically active IL-1 $\beta$. However, the exact mechanism by which mature IL- $1 \beta$ is released remains elusive.

The intrathecal injection of exogenous IL-1 $\beta$ is pronociceptive, ${ }^{29,37-39}$ resulting in both thermal and mechanical hypersensitivity. Two main mechanisms have been proposed to explain the contribution of IL-1 $\beta$ to neuropathic pain: first, direct action on neurons (either dorsal horn neurons or the 
central terminals of primary afferents); and second, indirect actions via activation of signaling pathways in immune cells. Studies indicate that IL- $1 \beta$ is able to increase the excitability of superficial dorsal horn neurons both in vitro ${ }^{29,39,40}$ and in vivo, ${ }^{37,41}$ as well as induce release of the primary afferent neurotransmitter Substance P. ${ }^{42}$ IL- $1 \beta$ is able to enhance glutamatergic synaptic transmission in lamina $\mathrm{I}^{29}$ and lamina II neurons. ${ }^{39,40}$ In addition, application of exogenous IL-1 $\beta$ to spinal cord slices in vitro is sufficient to induce a longterm potentiation (LTP) at C-fiber synapses with lamina I neurons. ${ }^{29}$ IL- $1 \beta$ is also able to reduce inhibitory synaptic transmission in vitro. ${ }^{39}$ Interestingly, despite the fact that IL- $1 \beta$ can directly enhance NMDA (N-methyl-D-aspartate) receptor phosphorylation, ${ }^{24,29,43}$ several recent studies suggest that the effects of IL- $1 \beta$ on neuronal excitability occur via an indirect mechanism..$^{29,40,41}$ Indeed, both behavioral ${ }^{29}$ and electrophysiological ${ }^{29,40,41}$ effects of IL-1 $\beta$ are absent following disruption of glial cell activity.

\section{TNF}

TNF (previously known as TNF $\alpha$ ) belongs to a superfamily of ligand/receptor proteins called the tumor necrosis factor/ tumor necrosis factor receptor superfamily proteins. TNF is an important proinflammatory cytokine for both inflammatory and immune processes, as well as in the generation of pain. TNF receptors are either constitutively expressed (TNFR1, p55-R) or inducible (TNFR2, p75-R) under inflammatory/injury conditions.

TNF is critical for the development of neuropathic pain, with a growing body of literature demonstrating that impairment of TNF signaling attenuates hypersensitivity in rodent models of neuropathy. The study of the role of TNF in neuropathic pain has been aided by a number of tools available to pharmacologically interfere with TNF signaling. These include anti-TNF antibodies (eg, infliximab), TNF soluble receptors (sTNFR), and recombinant TNFR-Fc fusion proteins (eg, etanercept). Intrathecal treatment with either sTNFR $^{20,44}$ or etanercept, ${ }^{45}$ beginning before peripheral nerve injury, is sufficient to prevent the development of neuropathic pain behaviors. Spinal delivery of sTNFR is able to prevent hypersensitivity induced by gp $120,{ }^{22}$ and intrathecal anti-TNF antibody is able to partially prevent the enhanced nociception induced by the chemotherapeutic agent vincristine. ${ }^{46}$ In addition, intrathecal administration of etanercept attenuates neuropathic pain behaviors in diabetic mice, ${ }^{47}$ and central pain induced by spinal cord injury in the rat. ${ }^{48}$ Interestingly, in the majority of studies pre-emptive treatment with antiTNF agents is required in order to inhibit pain behaviors, with delayed treatment ineffective, ${ }^{22,45,48}$ suggesting that TNF is an initiator of neuropathic pain. It also appears that the proinflammatory cytokines act synergistically under neuropathic pain conditions, as combined treatment using sTNFR with IL-1 ra demonstrates increased analgesic potency compared to sTNFR alone. ${ }^{20}$ One genetic study reported the same synergy in mice; TNF null mice develop normal pain behavior following peripheral nerve injury; however, mice null for both TNF and IL-1 $\beta$ fail to develop neuropathic hypersensitivity. ${ }^{49}$ Interestingly, transgenic mice that overexpress TNF in astrocytes exhibit significantly enhanced mechanical hypersensitivity compared to wild types following peripheral nerve injury. ${ }^{50}$

Under naive conditions, spinal expression of TNF is minimal, with rapid upregulation occurring following peripheral nerve injury. TNF is expressed by both glial cells ${ }^{51-53}$ and neurons. ${ }^{27,51,52}$ In addition, TNF receptors (both TNFR1 and 2) are also expressed by neurons and glia. ${ }^{29,51,52}$

The intrathecal injection of exogenous TNF is pronociceptive, ${ }^{29,39,54-56}$ resulting in both thermal and mechanical hypersensitivity. The use of proteins that selectively activate either TNFR1 or 2 suggests that spinal TNFR1 is the receptor primarily responsible for the pronociceptive effects of TNF under physiological conditions, whereas TNFR2 may begin to contribute following nerve injury, ${ }^{57}$ once injury-induced upregulation of the receptor has occurred. Similarly to studies with IL-1 $\beta$, spinal administration of TNF enhances dorsal horn neuronal responses in vivo ${ }^{37}$ and in vitro. ${ }^{29,39,55,56}$ In contrast, several studies have observed mixed or no changes to synaptic transmission following TNF application under naïve conditions. ${ }^{54,58}$ However, it appears that exogenous application of TNF to spinal cord slices is sufficient to induce LTP in lamina I neurons, ${ }^{29}$ and LTP induced by tetanic stimulation of the sciatic nerve is abolished in TNFR knockout mice. ${ }^{55}$ However, the ability of TNF to modulate synaptic transmission in the spinal dorsal horn may be mediated indirectly, via glial TNFRs. ${ }^{29}$ Indeed, blockade of TNF signaling significantly reduces injury associated reactivity of spinal glial cells. ${ }^{44,48}$ In particular, TNF stimulates an enhanced response state in spinal astrocytes, via increased phosphorylation of JNK (c-Jun N-terminal kinase) and release of the chemokine CCL2 ${ }^{59,60}$ (discussed in detail in the CCL2 section), which contributes to enhanced pain transmission during following peripheral nerve injury.

\section{Chemokines and spinal mechanisms in neuropathic pain}

Chemokines, or "chemotactic cytokines," are a family of small proteins that obtain their name from their first described 
function as mediators of leukocyte migration. First discovered in the late 1980s, the chemokines are now a large family of structurally and functionally similar molecules named according to the organization of cysteine residues on their $\mathrm{N}$-terminal region, and are divided into four subfamilies: $\mathrm{C}$, $\mathrm{CC}, \mathrm{CXC}$, and CX3C. Chemokines within each subclass have a promiscuous relationship with their receptors, of which there are over 20; as a result, receptor nomenclature is based on the subfamily of ligands it binds (eg, $\mathrm{CC}$ chemokines bind to $\mathrm{CC}$ receptors). The exception to this rule is the interaction between CX3CL1 and its receptor CX3CR1, which is a monogamous relationship. It is now well established that CNS cell types express chemokines and their receptors under both normal and pathological conditions, ${ }^{61}$ implying a role that goes beyond immune responses. ${ }^{62}$

\section{CX3CLI}

CX3CL1, also known as fractalkine, is the only member of the CX3C family of chemokines and was first described in 1997. ${ }^{63,64}$ The protein can exist in two forms, which mediate distinct biological actions: a membrane-tethered protein and a soluble chemokine domain. ${ }^{63,65}$ The latter is produced by the enzymatic cleavage of the chemokine domain of the former, by the metalloproteases ADAM10 and ADAM17, and the cysteine protease cathepsin S (CatS). ${ }^{4,66,67}$ Membrane-bound CX3CL1 serves as an adhesion molecule, promoting the firm adhesion of leukocytes without the activation of integrins, ${ }^{68}$ while soluble CX3CL1 is a potent chemoattractant for monocytes, natural killer cells, T cells, and B cells. ${ }^{65,69}$

Immunohistochemical studies have identified spinal cord neurons as constitutively expressing CX3CL1, ${ }^{70,71}$ with expression also observed in the cell bodies of sensory neurons in the dorsal root ganglia (DRG). ${ }^{72}$ However, while the neuronal location of CX3CL1 in the spinal cord was recently confirmed using CX3CL1-mCherry mice, ${ }^{73}$ the chemokine was not found in DRG cells, somehow questioning sensory neurons as a source of CX3CL1 outside the CNS. The receptor for CX3CL1, CX3CR1, is exclusively expressed by microglial cells within the spinal cord, and is extensively upregulated by nerve injury-induced microgliosis. $^{70,72,74}$ Figure 2 demonstrates the microglial expression of CX3CR1 in the dorsal horn using the CX3CR1-GFP (green fluorescent protein) mouse. ${ }^{75}$ In the dorsal horn of the spinal cord, all CX3CR1 expressing cells colocalize with the microglial cell marker Iba-1 (Figure 2). Therefore, the CX3CL1/CX3CR1 signaling pair has been proposed as a key mediator of neuronal-microglial communication during neuropathic pain states.
Under neuropathic pain conditions, neuronal CX3CL1 activates the microglial CX3CR1 receptor following proteolytic liberation of the chemokine extracellular domain. The enzyme responsible for CX3CL1 liberation is the lysosomal cysteine protease CatS, which is released by microglial cells in a $\mathrm{P} 2 \mathrm{X} 7$-mediated fashion. ${ }^{76} \mathrm{CX} 3 \mathrm{CL} 1$-mediated activation of microglial CX3CR1 results in phosphorylation of p38 mitogen-activated protein kinase ${ }^{66,74}$ and release of proinflammatory mediators, including IL-1 $\beta$, IL- 6 , and nitric oxide, ${ }^{77}$ that are able to sensitize neurons, thereby establishing a positive feedback mechanism that contributes to a chronic pain state. ${ }^{4}$ For example, CX3CL1 induces a hyper-responsive state in wide dynamic range neurons in the spinal cord. ${ }^{78}$ Indeed, intrathecal administration of either CatS inhibitors ${ }^{66,79,80}$ or antibodies against CX3CL1 or CX3CR1, ${ }^{66,74,80,81}$ attenuates behavioral hypersensitivity in models of neuropathic pain. Consistent with a pronociceptive effect of spinal CX3CL1, intrathecal injection of soluble CX3CL1 causes both mechanical and thermal hypersensitivity, ${ }^{66,74,77,81,82}$ while CX3CR1 knockout mice do not develop neuropathic pain behaviors following peripheral nerve injury ${ }^{83}$ Conversely, antinociceptive effects of CX3CL1 have been reported in the periphery (see Clark et $\mathrm{al}^{67}$ for detailed review).

While considering the suitability of CX3CL1 and CX3CR1 as targets for the development of analgesics, it is important to consider the protective effects of the interaction between these two proteins; in the brain, CX3CL1-CX3CR1 interaction is shown to be neuroprotective. ${ }^{84}$ Peripherally, this interaction is also critical for many homeostatic processes, including the survival of CX3CR $1^{\text {high }}$ monocyte/macrophages, wound healing, and cell transmigration for immune surveillance. Thus, when developing analgesics that target this interaction, a centrally acting compound, or an agent such as a CatS inhibitor that targets the shedding of soluble CX3CL1, leaving the membrane bound CX3CL1 intact, should be considered to minimize the occurrence of adverse effects.

\section{CCL2}

CCL2, also known as MCP-1 (monocyte chemoattractant protein-1), has been proposed to play a role in enhanced nociceptive transmission following peripheral injury. CCL2 was amongst the first human chemokines to be characterized, and belongs to a family of four other monocyte attracting chemokines that bear highly homologous structures. ${ }^{85}$

The expression of CCL2 in the peripheral nervous system has been studied extensively; injury-induced expression in DRG neurons has been demonstrated under neuropathic pain conditions (see Thacker et al, ${ }^{1}$ and Gao and $\mathrm{Ji}^{86}$ for detailed 

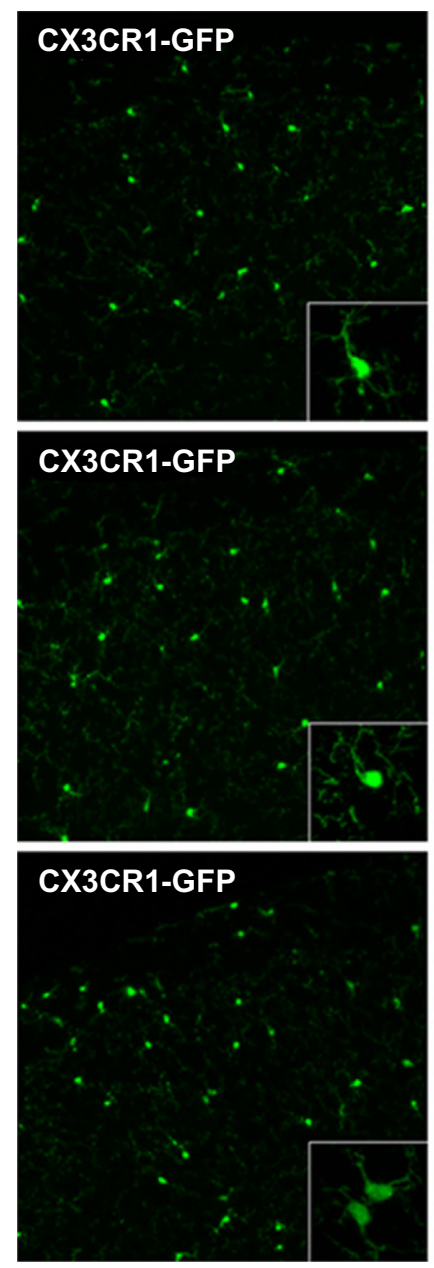
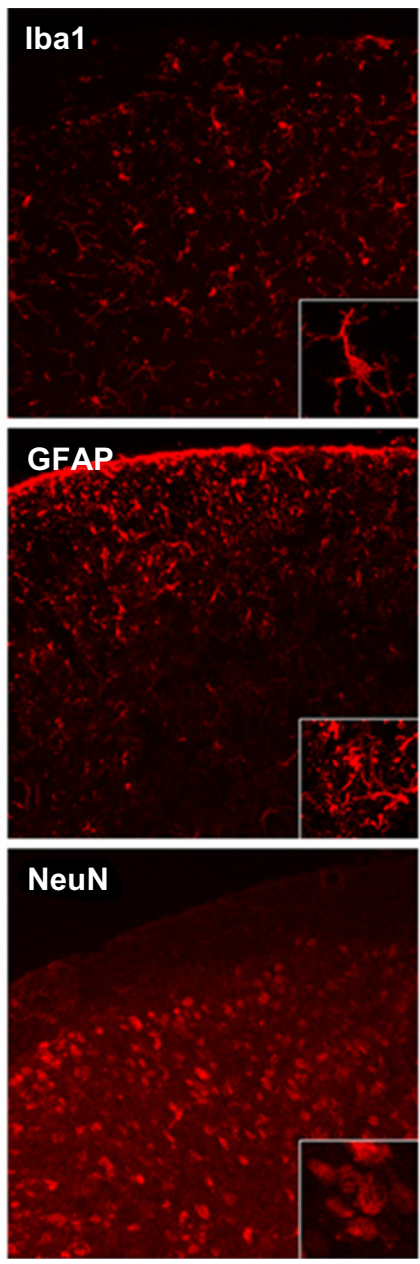
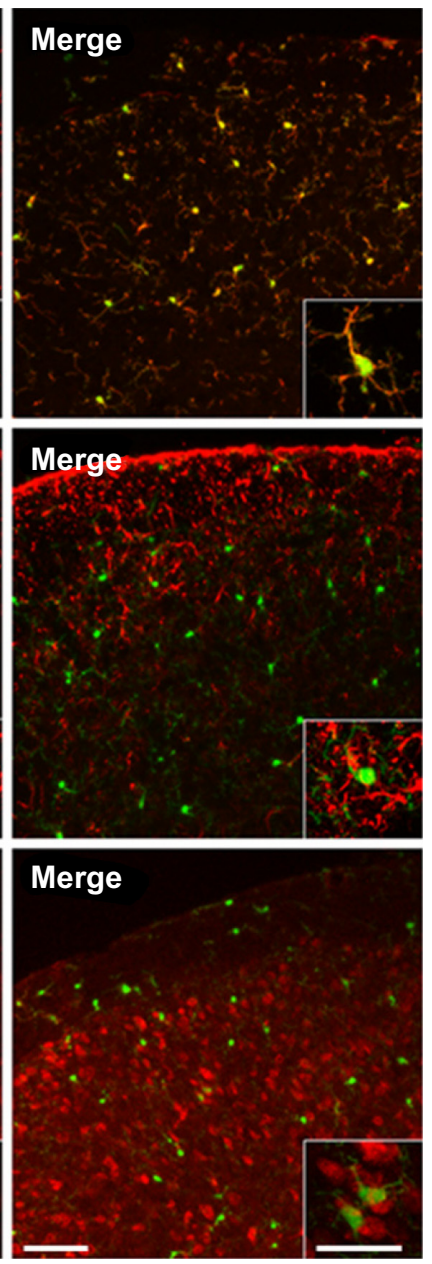

Figure 2 CX3CRI is expressed only by microglial cells in the spinal cord.

Notes: Immunohistochemical analysis conducted in our laboratory using a mouse in which the CX3CRI receptor is tagged with GFP75 reveals this protein is expressed exclusively by microglia in the dorsal horn of the spinal cord. Colocalization of CX3CRI is with a microglial cell marker (Ibal), but not an astrocytic (GFAP) or neuronal $(\mathrm{NeuN})$ marker. Scale bars are equal to $100 \mu \mathrm{m}$ and $30 \mu \mathrm{m}$ in high magnification inserts.

Abbreviations: CX3CRI, CX3C chemokine receptor I; GFAP, glial fibrillary acidic protein; GFP, green fluorescent protein; Iba I, ionized calcium-binding adapter molecule I; NeuN, neuronal nuclei.

review). Most studies show very low CCL2 expression in both the DRG and spinal cord under naïve conditions. However, one study suggests that CCL2 is constitutively expressed in primary afferent fibers, both in their cell bodies in the DRG and in the central terminals within the superficial lamina of the dorsal horn of the spinal cord. ${ }^{87}$ Indeed, following nerve injury, CCL2 expression in primary afferent terminals within the dorsal horn is dramatically increased., ${ }^{9,88}$ Under neuropathic conditions, CCL2 is released in an activity-dependent manner from primary afferent terminals within the dorsal horn. ${ }^{89,90}$ In addition, spinal astrocytes begin to express and release CCL2 following nerve injury. ${ }^{59}$ The spinal expression profile of the preferential receptor for CCL2, CCR2 remains heavily debated. CCR2 expression occurs in activated microglia following nerve injury, ${ }^{91}$ and in dorsal horn neurons under both naïve ${ }^{92}$ and nerve injury conditions, ${ }^{59}$ whereas astrocytic expression is observed following spinal cord injury. ${ }^{93}$ The above studies have utilized immunohistochemistry to examine CCR2 expression; however, the questionable specificity of the available antibodies has recently led to the development of a double CCR2/CCL2 reporter mouse. ${ }^{94}$ This transgenic mouse, in which CCL2/CCR2 interactions have been extensively characterized, suggests that, following a model of peripheral nerve demyelination (which results in the development of neuropathic hypersensitivity), there is virtually no spinal expression of either CCL2 or CCR2, ${ }^{94}$ calling into question earlier immunohistochemical studies.

Chemokine signaling, in particular the CCL2/CCR2 axis, is a key regulator of immune cell trafficking. Following peripheral nerve injury, leakage of the BSCB occurs, ${ }^{6,7}$ allowing the infiltration of peripheral immune cells into the spinal cord. Indeed, both macrophage ${ }^{6,8,9}$ and $\mathrm{T}$ lymphocyte $\mathrm{e}^{8,10-12}$ 
infiltration is observed in the dorsal horn following nerve injury and contributes to neuropathic hypersensitivity in rodent models. ${ }^{10,11} \mathrm{CCL} 2$ is critical in nerve injury-induced BSCB disruption ${ }^{6}$ as well as for the infiltration of CCR2 expressing bone marrow-derived macrophages into the spinal cord following peripheral nerve injury. ${ }^{9}$

While the precise location of CCR2 remains controversial, it is apparent that activation of this receptor by its primary ligand CCL2 plays a key role in the development of neuropathic pain. The intrathecal administration of CCL2 induces both mechanical and thermal hypersensitivity, , $9,87,89,91,95$ which is prevented by antibodies/antagonists directed against CCL2/CCR2 signaling. ${ }^{87,89}$ Importantly, CCR2 knockout mice exhibit significantly reduced pain behaviors following peripheral nerve injury. ${ }^{9,91}$ Pharmacological inhibition of CCL2/CCR2 signaling is also able to reverse established neuropathic pain behaviors; intrathecal delivery of either a CCL2 antibody ${ }^{59,89,96}$ or a CCR2 antagonist ${ }^{96,97}$ is able to reverse nerve injury- or chemotherapyinduced neuropathic hypersensitivity. Furthermore, systemic treatment with a CCR2 antagonist is also sufficient to reverse neuropathic pain behaviors in rodent models..$^{96,97}$ However, success in preclinical studies has not been followed by clinical success. Disappointingly, a recent clinical trial by AstraZeneca failed to demonstrate efficacy of a systemically administered CCR2 antagonist in patients with posttraumatic neuralgia. ${ }^{98}$

Two potential neuronal-glial signaling mechanisms have been suggested to underlie the role of CCL2/CCR2 in neuropathic pain. First, CCL2 released by primary afferent terminals ${ }^{89,90}$ may constitute a direct "activator" of microglia. In support of this hypothesis, intrathecally administered CCL2 results in extensive microglial activation, ${ }^{9,89}$ which is absent in CCR2 knockout mice. ${ }^{9}$ In addition, reversal of neuropathic pain by CCL2/CCR2 antibodies/antagonists is accompanied by reduced microglial activity in the dorsal horn, ${ }^{89,96}$ and CCR2 knockout mice exhibit significantly attenuated nerve injury-induced microgliosis compared to wild types. ${ }^{91}$ Secondly, astrocytic CCL2 may act via neuronal CCR2, directly inducing a sensitized state in dorsal horn neurons. Following peripheral nerve injury, release of CCL2 from astrocytes occurs in a JNK dependent manner ${ }^{59,60}$ resulting in phosphorylation of ERK in dorsal horn neurons,${ }^{59}$ an indicator of neuronal sensitization. Indeed, application of CCL2 to spinal cord slices is able to enhance glutamatergic synaptic transmission in lamina II neurons. ${ }^{59}$ In vivo the spinal application of a CCR2 antagonist is able to attenuate the activity of wide dynamic range neurons in neuropathic conditions, ${ }^{97}$ suggesting that spinal CCR2 may directly modulate neuronal activity.

\section{Anti-inflammatory cytokines and spinal mechanisms in neuropathic pain}

Immune responses involve a rapid production of proinflammatory cytokines, which serve to initiate the host's defense to pathogens and cellular damage. However, excessive inflammation may give rise to disturbances which are harmful to the host organism. Anti-inflammatory cytokines act to regulate the inflammatory process, limiting tissue damage and restoring homeostasis. In the case of neuropathic pain, the proinflammatory milieu of cytokines leads to excessive nociceptive transmission in the dorsal horn of the spinal cord. A dysregulation of the balance between pro- and antiinflammatory cytokines in the dorsal horn microenvironment appears to be causal in the chronicity of such pain states. Restoration of the cytokine balance may therefore represent a potential therapeutic avenue.

\section{IL- I0}

IL-10 is a potent anti-inflammatory cytokine and is essential for the regulation of immune responses. The antiinflammatory mechanisms of IL-10 have been extensively characterized, with dysregulation of IL-10 associated with inflammatory and autoimmune disorders. ${ }^{99-101}$ IL-10 was originally described as T helper 2 (Th2) cytokine, but is now known to be produced by many types of immune cells. Binding of IL-10 to the heterodimeric IL-10 receptor results in activation of the Janus kinase/signal transducer and activator of transcription (JAK/STAT) intracellular pathway, ultimately leading to anti-inflammatory activity. ${ }^{99}$ Of particular relevance for neuropathic pain states, IL-10 decreases nuclear factor kappa B activity, resulting in an attenuation of proinflammatory cytokine synthesis, including that of IL- $1 \beta$ and TNF.

Expression of both IL-10 and the IL-10 receptor are virtually undetectable in the naïve brain, but are enhanced in glial cells following pathology. ${ }^{102}$ Studies examining expression patterns within the spinal cord are limited. We have recently reported that IL-10 expression in T cells in the dorsal horn remains relatively unchanged following peripheral nerve injury, but can be boosted by glatiramer acetate treatment. ${ }^{12}$ However, both IL-10 and IL-10 receptor expression are upregulated in the spinal cord during pathology in the experimental autoimmune encephalomyelitis model of multiple sclerosis, most likely in glial cells. ${ }^{103}$ In humans, 
patients with a range of painful peripheral neuropathies exhibit decreased CSF levels of IL-10 compared to normal healthy controls, ${ }^{32,104}$ with one study observing an inverse correlation between IL-10 levels and patients' pain scores. ${ }^{32}$

A range of approaches has been utilized in order to enhance the expression of spinal IL-10 as a therapeutic strategy for the reversal of neuropathic pain. ${ }^{105}$ The direct intrathecal administration of recombinant IL-10 protein is able to transiently reverse (for a matter of hours) neuropathic pain behaviors induced by peripheral nerve injury, largely due to the short half-life of IL-10 within the CSF. ${ }^{17,106}$ Spinal delivery of either viral vectors ${ }^{17,107}$ or naked plasmid DNA ${ }^{18,19,108}$ encoding IL-10 protein results in a longer-lasting reversal (lasting several weeks) of both mechanical and thermal hypersensitivity following nerve injury, with enhanced levels of IL-10 protein correlating with reduced levels of IL- $1 \beta$ in the CSF. ${ }^{17}$ Likewise, intrathecal polymer based IL-10 delivery systems further prolong (weeks to months) the reversal of neuropathic pain behaviors achievable. ${ }^{18,106,109}$ Interestingly, we have recently reported that following a peripheral nerve injury treatment with glatiramer acetate (also known as Copolymer 1) reverses established neuropathic hypersensitivity and that this correlates with enhanced expression levels of IL-10 within both $\mathrm{T}$ cells and other cells within the spinal cord, ${ }^{12}$ suggesting that modulation of the Th1/Th2 balance within the spinal cord may be a potential therapeutic strategy for neuropathic pain.

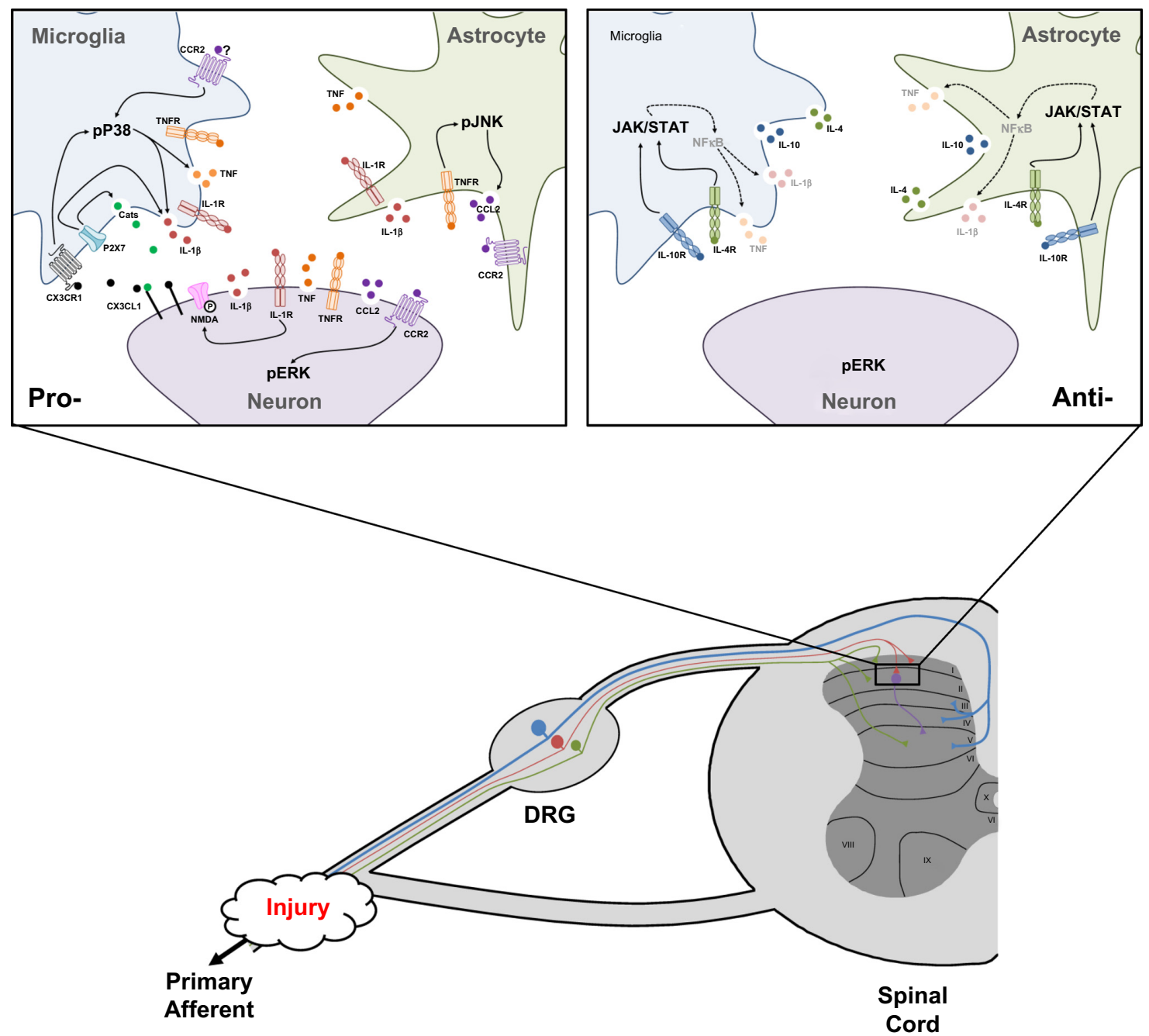

Figure 3 Schematic representation of spinal cytokine/chemokine signaling mechanisms which contribute to enhanced nociceptive transmission following peripheral nerve injury.

Notes: Primary afferent fibers (A $\beta$ [blue], A $\delta$ [green] and $C$ [red]) transmit signals from the periphery, through the DRG to the dorsal horn of the spinal cord. Following damage to a peripheral nerve, a number of cytokine/chemokine signaling systems exhibit plastic changes at the first synapse in the pain pathway.

Abbreviations: $A \beta, A$ beta fiber; $A \delta$, A delta fiber; DRG, dorsal root ganglia; JAK/STAT, Janus kinase/signal transducer and activator of transcription; IL, interleukin; NMDA, N-methyl-D-aspartate; CCL2, chemokine ligand 2; TNF, tumor necrosis factor; NFאB, nuclear factor kappa B; TNFR, tumor necrosis factor receptor; CX3CRI, CX3C chemokine receptor I; CX3CLI, CX3C chemokine ligand I; CatS, cathepsin S; CCR2, chemokine receptor type 2; R, receptor; JNK, c-Jun N-terminal kinase; ERK, extracellular signal-regulated kinase; p38, p38 mitogen-activated protein kinase. 


\section{IL-4}

IL-4 is a prototypical anti-inflammatory cytokine that functions as a potent regulator of immunity and is secreted by a number of immune cell types. IL-4 is best characterized for promoting Th2 skewing of T cells, as well as being an archetypal inducer of M2 "alternative" macrophage phenotype. IL- 4 signaling is mediated by the IL- $4 \mathrm{R} \alpha$-chain, which forms either the type 1 (hematopoietic cells) or type 2 (nonhematopoietic cells) receptor complex. ${ }^{110,111}$ Binding of IL-4 to either receptor complex results in activation of the JAK/STAT intracellular pathway. ${ }^{10,111}$

Several studies have suggested that IL-4, derived from peripheral immune cells, is antinociceptive in inflammatory pain models. However, the role of IL-4 in models of neuropathic pain has been rarely investigated. Lack of IL-4 leads to a mechanical hypersensitivity; IL-4 knockout mice exhibit significantly reduced mechanical withdrawal thresholds under naïve conditions compared to wild types. ${ }^{12}$ Following a peripheral nerve injury, no changes in the mechanical withdrawal threshold are observed in IL-4 null mice; however, this is primarily due to very low mechanical withdrawal thresholds at baseline, with further reduction exceeding the sensitivity of behavioral tests. ${ }^{12}$ Interestingly, IL-4 null mice show an enhanced upregulation of spinal proinflammatory cytokines following nerve injury compared to wild-type mice, despite exhibiting comparable levels under naïve conditions. ${ }^{11}$ In addition, we have recently reported that enhanced expression levels of IL-4 within the spinal cord correlate with reversal of neuropathic hypersensitivity following treatment with glatiramer acetate. ${ }^{12} \mathrm{In}$ humans, decreased CSF levels of IL-4 have been reported in CRPS patients. ${ }^{104}$ Therefore, it is tempting to speculate that a therapeutic strategy to induce overexpression of spinal IL-4 may be efficacious for the treatment of neuropathic pain, as is the case for IL-10.

\section{Conclusion}

We have considered a selected number of cytokines and chemokines which play pro- or antinociceptive roles at the first pain synapse under neuropathic pain conditions. Cytokines and chemokines are released by neurons, microglia, astrocytes, macrophages and $T$ cells and activate pain neurons directly and via activation of non-neuronal cells, depending on the expression of their receptors (Figure 3). Indeed, inhibition of proinflammatory cytokines and induction of anti-inflammatory cytokine expression results in antinociception. The future task will be the translation of this preclinical evidence in effective treatments for neuropathic pain in patients.

\section{Acknowledgments}

This work was supported by the Wellcome Trust (Wellcome Trust Flexible Travel Fellowship to AKC) and a BBSRC CASE studentship (EAO, MM). MM would like to acknowledge Arthritis Research UK for continued support.

\section{Disclosure}

The authors declare no conflicts of interest in this work.

\section{References}

1. Thacker MA, Clark AK, Marchand F, McMahon SB. Pathophysiology of peripheral neuropathic pain: immune cells and molecules. Anesth Analg. 2007;105(3):838-847.

2. Austin PJ, Moalem-Taylor G. The neuro-immune balance in neuropathic pain: involvement of inflammatory immune cells, immune-like glial cells and cytokines. J Neuroimmunol. 2010;229(1-2):26-50.

3. McMahon SB, Malcangio M. Current challenges in glia-pain biology. Neuron. 2009;64(1):46-54

4. Clark AK, Malcangio M. Microglial signalling mechanisms: Cathepsin S and Fractalkine. Exp Neurol. 2012;234(2):283-292.

5. Hansen RR, Malcangio M. Astrocytes-Multitaskers in chronic pain. Eur J Pharmacol. 2013;716(1-3):120-128.

6. Echeverry S, Shi XQ, Rivest S, Zhang J. Peripheral nerve injury alters blood-spinal cord barrier functional and molecular integrity through a selective inflammatory pathway. J Neurosci. 2011;31(30): 10819-10828.

7. Beggs S, Liu XJ, Kwan C, Salter MW. Peripheral nerve injury and TRPV1-expressing primary afferent $\mathrm{C}$-fibers cause opening of the blood-brain barrier. Mol Pain. 2010;6:74.

8. Sweitzer SM, Hickey WF, Rutkowski MD, Pahl JL, DeLeo JA. Focal peripheral nerve injury induces leukocyte trafficking into the central nervous system: potential relationship to neuropathic pain. Pain 2002;100(1-2):163-170.

9. Zhang J, Shi XQ, Echeverry S, Mogil JS, De Koninck Y, Rivest $\mathrm{S}$. Expression of CCR2 in both resident and bone marrow-derived microglia plays a critical role in neuropathic pain. $J$ Neurosci. 2007;27(45):12396-12406.

10. Cao L, DeLeo JA. CNS-infiltrating CD4+ T lymphocytes contribute to murine spinal nerve transection-induced neuropathic pain. Eur $J$ Immunol. 2008;38(2):448-458.

11. Costigan M, Moss A, Latremoliere A, et al. T-cell infiltration and signaling in the adult dorsal spinal cord is a major contributor to neuropathic pain-like hypersensitivity. J Neurosci. 2009;29(46):14415-14422.

12. Leger T, Grist J, D'Acquisto F, Clark AK, Malcangio M. Glatiramer acetate attenuates neuropathic allodynia through modulation of adaptive immune cells. J Neuroimmunol. 2011;234(1-2):19-26.

13. Dinarello CA. Historical insights into cytokines. Eur J Immunol. 2007;37 Suppl 1:S34-S45.

14. Honore P, Wade CL, Zhong C, et al. Interleukin-1alphabeta gene-deficient mice show reduced nociceptive sensitivity in models of inflammatory and neuropathic pain but not post-operative pain. Behav Brain Res. 2006;167(2):355-364.

15. Wolf G, Gabay E, Tal M, Yirmiya R, Shavit Y. Genetic impairment of interleukin-1 signaling attenuates neuropathic pain, autotomy, and spontaneous ectopic neuronal activity, following nerve injury in mice. Pain. 2006;120(3):315-324.

16. Gabay E, Wolf G, Shavit Y, Yirmiya R, Tal M. Chronic blockade of interleukin-1 (IL-1) prevents and attenuates neuropathic pain behavior and spontaneous ectopic neuronal activity following nerve injury. Eur J Pain. 2011;15(3):242-248.

17. Milligan ED, Langer SJ, Sloane EM, et al. Controlling pathological pain by adenovirally driven spinal production of the anti-inflammatory cytokine, interleukin-10. Eur J Neurosci. 2005;21(8):2136-2148. 
18. Milligan ED, Soderquist RG, Malone SM, et al. Intrathecal polymerbased interleukin-10 gene delivery for neuropathic pain. Neuron Glia Biol. 2006;2(4):293-308.

19. Ledeboer A, Jekich BM, Sloane EM, et al. Intrathecal interleukin-10 gene therapy attenuates paclitaxel-induced mechanical allodynia and proinflammatory cytokine expression in dorsal root ganglia in rats. Brain Behav Immun. 2007;21(5):686-698.

20. Sweitzer S, Martin D, DeLeo JA. Intrathecal interleukin-1 receptor antagonist in combination with soluble tumor necrosis factor receptor exhibits an anti-allodynic action in a rat model of neuropathic pain. Neuroscience. 2001;103(2):529-539.

21. Clark AK, D’Aquisto F, Gentry C, Marchand F, McMahon SB, Malcangio M. Rapid co-release of interleukin 1beta and caspase 1 in spinal cord inflammation. J Neurochem. 2006;99(3):868-880.

22. Milligan ED, O’Connor KA, Nguyen KT, et al. Intrathecal HIV-1 envelope glycoprotein gp120 induces enhanced pain states mediated by spinal cord proinflammatory cytokines. J Neurosci. 2001;21(8):2808-2819.

23. Felts PA, Woolston AM, Fernando HB, et al. Inflammation and primary demyelination induced by the intraspinal injection of lipopolysaccharide. Brain. 2005;128(Pt 7):1649-1666.

24. Zhang RX, Li A, Liu B, et al. IL-1ra alleviates inflammatory hyperalgesia through preventing phosphorylation of NMDA receptor NR-1 subunit in rats. Pain. 2008;135(3):232-239.

25. Liao YH, Zhang GH, Jia D, et al. Spinal astrocytic activation contributes to mechanical allodynia in a mouse model of type 2 diabetes. Brain Res. 2011;1368:324-335.

26. Ji XT, Qian NS, Zhang T, et al. Spinal astrocytic activation contributes to mechanical allodynia in a rat chemotherapy-induced neuropathic pain model. PLoS One. 2013;8(4):e60733.

27. DeLeo JA, Colburn RW, Rickman AJ. Cytokine and growth factor immunohistochemical spinal profiles in two animal models of mononeuropathy. Brain Res. 1997;759(1):50-57.

28. Sweitzer SM, Colburn RW, Rutkowski M, DeLeo JA. Acute peripheral inflammation induces moderate glial activation and spinal IL-1beta expression that correlates with pain behavior in the rat. Brain Res. 1999;829(1-2):209-221.

29. Gruber-Schoffnegger D, Drdla-Schutting R, Hönigsperger C, Wunderbaldinger G, Gassner M, Sandkühler J. Induction of thermal hyperalgesia and synaptic long-term potentiation in the spinal cord lamina I by TNF- $\alpha$ and IL- $1 \beta$ is mediated by glial cells. J Neurosci. 2013;33(15):6540-6551.

30. Whitehead KJ, Smith CG, Delaney SA, et al. Dynamic regulation of spinal pro-inflammatory cytokine release in the rat in vivo following peripheral nerve injury. Brain Behav Immun. 2010;24(4):569-576.

31. Alexander GM, van Rijn MA, van Hilten JJ, Perreault MJ, Schwartzman RJ. Changes in cerebrospinal fluid levels of pro-inflammatory cytokines in CRPS. Pain. 2005;116(3):213-219.

32. Backonja MM, Coe CL, Muller DA, Schell K. Altered cytokine levels in the blood and cerebrospinal fluid of chronic pain patients. J Neuroimmunol. 2008;195(1-2):157-163.

33. Martinon F, Burns K, Tschopp J. The inflammasome: a molecular platform triggering activation of inflammatory caspases and processing of proIL-beta. Mol Cell. 2002;10(2):417-426.

34. Thornberry NA, Bull HG, Calaycay JR, et al. A novel heterodimeric cysteine protease is required for interleukin-1 beta processing in monocytes. Nature. 1992;356(6372):768-774.

35. Ellis A, Bennett DL. Investigating the inflammasome within microglia and its modulation following nerve injury [abstract]. Ellis A, Bennett DL. International Association for the Study of Pain, 13th World Congress on Pain, Milan, Italy; 2012:PH373.

36. Kawasaki Y, Xu ZZ, Wang X, et al. Distinct roles of matrix metalloproteases in the early- and late-phase development of neuropathic pain. Nat Med. 2008;14(3):331-336.

37. Reeve AJ, Patel S, Fox A, Walker K, Urban L. Intrathecally administered endotoxin or cytokines produce allodynia, hyperalgesia and changes in spinal cord neuronal responses to nociceptive stimuli in the rat. Eur J Pain. 2000;4(3):247-257.
38. Sung CS, Wen ZH, Chang WK, et al. Intrathecal interleukin-1beta administration induces thermal hyperalgesia by activating inducible nitric oxide synthase expression in the rat spinal cord. Brain Res. 2004;1015(1-2):145-153

39. Kawasaki Y, Zhang L, Cheng JK, Ji RR. Cytokine mechanisms of central sensitization: distinct and overlapping role of interleukin-1beta, interleukin-6, and tumor necrosis factor-alpha in regulating synaptic and neuronal activity in the superficial spinal cord. J Neurosci. 2008;28(20): 5189-5194.

40. Liu T, Jiang CY, Fujita T, Luo SW, Kumamoto E. Enhancement by interleukin-1 $\beta$ of AMPA and NMDA receptor-mediated currents in adult rat spinal superficial dorsal horn neurons. Mol Pain. 2013; 9:16.

41. Constandil L, Hernández A, Pelissier T, et al. Effect of interleukin-1beta on spinal cord nociceptive transmission of normal and monoarthritic rats after disruption of glial function. Arthritis Res Ther. 2009;11(4): R105.

42. Malcangio M, Bowery NG, Flower RJ, Perretti M. Effect of interleukin-1 beta on the release of substance P from rat isolated spinal cord. Eur $J$ Pharmacol. 1996;299(1-3):113-118.

43. Wei F, Guo W, Zou S, Ren K, Dubner R. Supraspinal glial-neuronal interactions contribute to descending pain facilitation. J Neurosci. 2008;28(42):10482-10495.

44. Ishikawa T, Miyagi M, Kamoda H, et al. Differences between tumor necrosis factor- $\alpha$ receptors types 1 and 2 in the modulation of spinal glial cell activation and mechanical allodynia in a rat sciatic nerve injury model. Spine (Phila Pa 1976). 2013;38(1):11-16.

45. Svensson CI, Schäfers M, Jones TL, Powell H, Sorkin LS. Spinal blockade of TNF blocks spinal nerve ligation-induced increases in spinal P-p38. Neurosci Lett. 2005;379(3):209-213.

46. Kiguchi N, Maeda T, Kobayashi Y, Kishioka S. Up-regulation of tumor necrosis factor-alpha in spinal cord contributes to vincristineinduced mechanical allodynia in mice. Neurosci Lett. 2008;445(2): 140-143.

47. Dogrul A, Gul H, Yesilyurt O, Ulas UH, Yildiz O. Systemic and spinal administration of etanercept, a tumor necrosis factor alpha inhibitor, blocks tactile allodynia in diabetic mice. Acta Diabetol. 2011;48(2): 135-142.

48. Marchand F, Tsantoulas C, Singh D, et al. Effects of Etanercept and Minocycline in a rat model of spinal cord injury. Eur J Pain. 2009;13(7): 673-681.

49. Nadeau S, Filali M, Zhang J, et al. Functional recovery after peripheral nerve injury is dependent on the pro-inflammatory cytokines IL-1 $\beta$ and TNF: implications for neuropathic pain. J Neurosci. 2011;31(35): 12533-12542.

50. DeLeo JA, Rutkowski MD, Stalder AK, Campbell IL. Transgenic expression of TNF by astrocytes increases mechanical allodynia in a mouse neuropathy model. Neuroreport. 2000;11(3):599-602.

51. Xu JT, Xin WJ, Zang Y, Wu CY, Liu XG. The role of tumor necrosis factor-alpha in the neuropathic pain induced by Lumbar 5 ventral root transection in rat. Pain. 2006;123(3):306-321.

52. Ohtori S, Takahashi K, Moriya H, Myers RR. TNF-alpha and TNF-alpha receptor type 1 upregulation in glia and neurons after peripheral nerve injury: studies in murine DRG and spinal cord. Spine (Phila Pa 1976). 2004;29(10):1082-1088.

53. Hao S, Mata M, Glorioso JC, Fink DJ. Gene transfer to interfere with TNFalpha signaling in neuropathic pain. Gene Ther. 2007;14(13): 1010-1016.

54. Youn DH, Wang H, Jeong SJ. Exogenous tumor necrosis factor-alpha rapidly alters synaptic and sensory transmission in the adult rat spinal cord dorsal horn. J Neurosci Res. 2008;86(13):2867-2875.

55. Park CK, Lü N, Xu ZZ, Liu T, Serhan CN, Ji RR. Resolving TRPV1and TNF- $\alpha$-mediated spinal cord synaptic plasticity and inflammatory pain with neuroprotectin D1. J Neurosci. 2011;31(42):15072-15085.

56. Zhang L, Berta T, Xu ZZ, Liu T, Park JY, Ji RR. TNF- $\alpha$ contributes to spinal cord synaptic plasticity and inflammatory pain: distinct role of TNF receptor subtypes 1 and 2. Pain. 2011;152(2):419-427. 
57. Schäfers M, Sommer C, Geis C, Hagenacker T, Vandenabeele P, Sorkin LS. Selective stimulation of either tumor necrosis factor receptor differentially induces pain behavior in vivo and ectopic activity in sensory neurons in vitro. Neuroscience. 2008;157(2):414-423.

58. Liu YL, Zhou LJ, Hu NW, et al. Tumor necrosis factor-alpha induces long-term potentiation of C-fiber evoked field potentials in spinal dorsal horn in rats with nerve injury: the role of NF-kappa B, JNK and p38 MAPK. Neuropharmacology. 2007;52(3):708-715.

59. Gao YJ, Zhang L, Samad OA, et al. JNK-induced MCP-1 production in spinal cord astrocytes contributes to central sensitization and neuropathic pain. J Neurosci. 2009;29(13):4096-4108.

60. Gao YJ, Zhang L, Ji RR. Spinal injection of TNF- $\alpha$-activated astrocytes produces persistent pain symptom mechanical allodynia by releasing monocyte chemoattractant protein-1. Glia. 2010;58(15):1871-1880.

61. Bajetto A, Bonavia R, Barbero S, Schettini G. Characterization of chemokines and their receptors in the central nervous system: physiopathological implications. J Neurochem. 2002;82(6):1311-1329.

62. Réaux-Le Goazigo A, Van Steenwinckel J, Rostène W, Mélik Parsadaniantz S. Current status of chemokines in the adult CNS. Prog Neurobiol. 2013;104:67-92.

63. Bazan JF, Bacon KB, Hardiman G, et al. A new class of membrane-bound chemokine with a CX3C motif. Nature. 1997;385(6617):640-644.

64. Pan Y, Lloyd C, Zhou H, et al. Neurotactin, a membrane-anchored chemokine upregulated in brain inflammation. Nature. 1997;387(6633): 611-617.

65. Imai $\mathrm{T}$, Hieshima $\mathrm{K}$, Haskell $\mathrm{C}$, et al. Identification and molecular characterization of fractalkine receptor CX3CR1, which mediates both leukocyte migration and adhesion. Cell. 1997;91(4):521-530.

66. Clark AK, Yip PK, Grist J, et al. Inhibition of spinal microglial cathepsin S for the reversal of neuropathic pain. Proc Natl Acad Sci USA. 2007;104(25):10655-10660.

67. Clark AK, Staniland AA, Malcangio M. Fractalkine/CX3CR1 signalling in chronic pain and inflammation. Curr Pharm Biotechnol. 2011;12(10):1707-1714.

68. Fong AM, Robinson LA, Steeber DA, et al. Fractalkine and CX3CR1 mediate a novel mechanism of leukocyte capture, firm adhesion, and activation under physiologic flow. $J$ Exp Med. 1998;188(8): 1413-1419.

69. Corcione A, Ferretti E, Bertolotto M, et al. CX3CR1 is expressed by human B lymphocytes and mediates [corrected] CX3CL1 driven chemotaxis of tonsil centrocytes. PLoS One. 2009;4(12):e8485.

70. Lindia JA, McGowan E, Jochnowitz N, Abbadie C. Induction of CX3CL1 expression in astrocytes and CX3CR1 in microglia in the spinal cord of a rat model of neuropathic pain. J Pain. 2005;6(7):434-438.

71. Clark AK, Yip PK, Malcangio M. The liberation of fractalkine in the dorsal horn requires microglial cathepsin S. J Neurosci. 2009;29(21): 6945-6954.

72. Verge GM, Milligan ED, Maier SF, Watkins LR, Naeve GS, Foster AC. Fractalkine (CX3CL1) and fractalkine receptor (CX3CR1) distribution in spinal cord and dorsal root ganglia under basal and neuropathic pain conditions. Eur J Neurosci. 2004;20(5):1150-1160.

73. Kim KW, Vallon-Eberhard A, Zigmond E, et al. In vivo structure/function and expression analysis of the $\mathrm{CX} 3 \mathrm{C}$ chemokine fractalkine. Blood. 2011;118(22):e156-e167.

74. Zhuang ZY, Kawasaki Y, Tan PH, Wen YR, Huang J, Ji RR. Role of the CX3CR1/p38 MAPK pathway in spinal microglia for the development of neuropathic pain following nerve injury-induced cleavage of fractalkine. Brain Behav Immun. 2007;21(5):642-651.

75. Jung S, Aliberti J, Graemmel P, et al. Analysis of fractalkine receptor CX(3)CR1 function by targeted deletion and green fluorescent protein reporter gene insertion. Mol Cell Biol. 2000;20(11):4106-4114.

76. Clark AK, Wodarski R, Guida F, Sasso O, Malcangio M. Cathepsin $\mathrm{S}$ release from primary cultured microglia is regulated by the P2X7 receptor. Glia. 2010;58(14):1710-1726.

77. Milligan E, Zapata V, Schoeniger D, et al. An initial investigation of spinal mechanisms underlying pain enhancement induced by fractalkine, a neuronally released chemokine. Eur J Neurosci. 2005;22(11): 2775-2782.
78. Owolabi SA, Saab CY. Fractalkine and minocycline alter neuronal activity in the spinal cord dorsal horn. FEBS Lett. 2006;580(18): 4306-4310.

79. Clark AK, Marchand F, D'Auria M et al. Cathepsin S inhibiton attenuates neuropathic pain and microglial response associated with spinal cord injury. Open Pain J. 2010;3:117-122.

80. Clark AK, Grist J, Al-Kashi A, Perretti M, Malcangio M. Spinal cathepsin $\mathrm{S}$ and fractalkine contribute to chronic pain in the collageninduced arthritis model. Arthritis Rheum. 2012;64(6):2038-2047.

81. Milligan ED, Zapata V, Chacur M, et al. Evidence that exogenous and endogenous fractalkine can induce spinal nociceptive facilitation in rats. Eur J Neurosci. 2004;20(9):2294-2302.

82. Sun JL, Xiao C, Lu B, et al. CX3CL1/CX3CR1 regulates nerve injuryinduced pain hypersensitivity through the ERK5 signaling pathway. J Neurosci Res. 2013;91(4):545-553.

83. Staniland AA, Clark AK, Wodarski R, et al. Reduced inflammatory and neuropathic pain and decreased spinal microglial response in fractalkine receptor (CX3CR1) knockout mice. J Neurochem. 2010;114(4): $1143-1157$.

84. Wolf Y, Yona S, Kim KW, Jung S. Microglia, seen from the CX3CR1 angle. Front Cell Neurosci. 2013;7:26.

85. Van Coillie E, Van Damme J, Opdenakker G. The MCP/eotaxin subfamily of CC chemokines. Cytokine Growth Factor Rev. 1999;10(1): $61-86$

86. Gao YJ, Ji RR. Chemokines, neuronal-glial interactions, and central processing of neuropathic pain. Pharmacol Ther. 2010;126(1):56-68.

87. Dansereau MA, Gosselin RD, Pohl M, et al. Spinal CCL2 pronociceptive action is no longer effective in CCR2 receptor antagonist-treated rats. J Neurochem. 2008;106(2):757-769.

88. Jeon SM, Lee KM, Cho HJ. Expression of monocyte chemoattractant protein-1 in rat dorsal root ganglia and spinal cord in experimental models of neuropathic pain. Brain Res. 2009;1251:103-111.

89. Thacker MA, Clark AK, Bishop T, et al. CCL2 is a key mediator of microglia activation in neuropathic pain states. Eur J Pain. 2009;13(3): 263-272.

90. Van Steenwinckel J, Reaux-Le Goazigo A, Pommier B et al. CCL2 released from neuronal synaptic vesicles in the spinal cord is a major mediator of local inflammation and pain after peripheral nerve injury. J Neurosci. 2011;31(15):5865-5875.

91. Abbadie C, Lindia JA, Cumiskey AM, et al. Impaired neuropathic pain responses in mice lacking the chemokine receptor CCR2. Proc Natl Acad Sci U S A. 2003;100(13):7947-7952.

92. Gosselin RD, Varela C, Banisadr G, et al. Constitutive expression of CCR2 chemokine receptor and inhibition by MCP-1/CCL2 of GABAinduced currents in spinal cord neurones. $J$ Neurochem. 2005;95(4): 1023-1034.

93. Knerlich-Lukoschus F, Juraschek M, Blömer U, Lucius R, Mehdorn HM, Held-Feindt J. Force-dependent development of neuropathic central pain and time-related CCL2/CCR2 expression after graded spinal cord contusion injuries of the rat. J Neurotrauma. 2008;25(5):427-448.

94. Jung $\mathrm{H}$, Bhangoo $\mathrm{S}$, Banisadr $\mathrm{G}$, et al. Visualization of chemokine receptor activation in transgenic mice reveals peripheral activation of CCR2 receptors in states of neuropathic pain. J Neurosci. 2009;29(25): 8051-8062.

95. Tanaka T, Minami M, Nakagawa T, Satoh M. Enhanced production of monocyte chemoattractant protein-1 in the dorsal root ganglia in a rat model of neuropathic pain: possible involvement in the development of neuropathic pain. Neurosci Res. 2004;48(4):463-469.

96. Pevida M, Lastra A, Hidalgo A, Baamonde A, Menéndez L. Spinal CCL2 and microglial activation are involved in paclitaxel-evoked cold hyperalgesia. Brain Res Bull. 2013;95:21-27.

97. Serrano A, Paré M, McIntosh F, et al. Blocking spinal CCR2 with AZ889 reversed hyperalgesia in a model of neuropathic pain. Mol Pain. 2010;6:90.

98. Kalliomäki J, Attal N, Jonzon B, et al; AZD2423 PTN Study Group. A randomized, double-blind, placebo-controlled trial of a chemokine receptor 2 (CCR2) antagonist in posttraumatic neuralgia. Pain. 2013;154(5):761-767. 
99. Mosser DM, Zhang X. Interleukin-10: new perspectives on an old cytokine. Immunol Rev. 2008;226:205-218.

100. Saraiva M, O'Garra A. The regulation of IL-10 production by immune cells. Nat Rev Immunol. 2010;10(3):170-181.

101. Iyer SS, Cheng G. Role of interleukin 10 transcriptional regulation in inflammation and autoimmune disease. Crit Rev Immunol. 2012;32(1):23-63.

102. Hulshof S, Montagne L, De Groot CJ, Van Der Valk P. Cellular localization and expression patterns of interleukin-10, interleukin-4, and their receptors in multiple sclerosis lesions. Glia. 2002;38(1):24-35.

103. Ledeboer A, Wierinckx A, Bol JG, et al. Regional and temporal expression patterns of interleukin-10, interleukin-10 receptor and adhesion molecules in the rat spinal cord during chronic relapsing EAE. J Neuroimmunol. 2003;136(1-2):94-103.

104. Alexander GM, Perreault MJ, Reichenberger ER, Schwartzman RJ. Changes in immune and glial markers in the CSF of patients with Complex Regional Pain Syndrome. Brain Behav Immun. 2007;21(5): 668-676.

105. Milligan ED, Penzkover KR, Soderquist RG, Mahoney MJ. Spinal interleukin-10 therapy to treat peripheral neuropathic pain. Neuromodulation. 2012;15(6):520-526; discussion 526-.
106. Soderquist RG, Milligan ED, Harrison JA, et al. PEGylation of interleukin-10 for the mitigation of enhanced pain states. J Biomed Mater Res A. 2010;93(3):1169-1179.

107. Milligan ED, Sloane EM, Langer SJ, et al. Controlling neuropathic pain by adeno-associated virus driven production of the anti-inflammatory cytokine, interleukin-10. Mol Pain. 2005;1:9.

108. Milligan ED, Sloane EM, Langer SJ, et al. Repeated intrathecal injections of plasmid DNA encoding interleukin-10 produce prolonged reversal of neuropathic pain. Pain. 2006;126(1-3):294-308.

109. Soderquist RG, Sloane EM, Loram LC, et al. Release of plasmid DNA-encoding IL-10 from PLGA microparticles facilitates longterm reversal of neuropathic pain following a single intrathecal administration. Pharm Res. 2010;27(5):841-854.

110. Gadani SP, Cronk JC, Norris GT, Kipnis J. IL-4 in the brain: a cytokine to remember. J Immunol. 2012;189(9):4213-4219.

111. Jiang H, Harris MB, Rothman P. IL-4/IL-13 signaling beyond JAK/ STAT. J Allergy Clin Immunol. 2000;105(6 Pt 1):1063-1070.

112. Üçeyler N, Topuzoğlu T, Schiesser P, Hahnenkamp S, Sommer C. IL-4 deficiency is associated with mechanical hypersensitivity in mice. PLoS One. 2011;6(12):e28205.
Journal of Pain Research

\section{Publish your work in this journal}

The Journal of Pain Research is an international, peer-reviewed, open access, online journal that welcomes laboratory and clinical findings in the fields of pain research and the prevention and management of pain. Original research, reviews, symposium reports, hypothesis formation and commentaries are all considered for publication.

\section{Dovepress}

The manuscript management system is completely online and includes a very quick and fair peer-review system, which is all easy to use. Visit http://www.dovepress.com/testimonials.php to read real quotes from published authors. 